\title{
Bottom-Up Preparation Techniques for Nanocrystals of Lipophilic Drugs
}

\author{
Hans de Waard • Henderik W. Frijlink • Wouter L. J. Hinrichs
}

Received: 7 October 2010 / Accepted: 8 November 2010 / Published online: 18 November 2010

(C) The Author(s) 2010. This article is published with open access at Springerlink.com

KEY WORDS bottom-up · large-scale production . nanocrystal · solubility

\section{INTRODUCTION}

Modern drug discovery techniques result in the development of an increasing number of highly lipophilic drug candidates. Their lipophilicity results in a slow dissolution of these drugs in the aqueous gastro-intestinal fluids, resulting in a poor bioavailability. Over the past few decades, many strategies have been developed to increase the dissolution rate of these types of drugs. The application of drug nanocrystals is one technique. The dissolution rate of drugs from nanocrystals is increased due to an increase of the saturation concentration around these particles (Kelvin law) and an increase of the surface area available for dissolution.

Preparation techniques for drug nanocrystals can be divided into top-down and bottom-up techniques. Topdown techniques are based on size reduction of relatively large particles into smaller particles, whereas bottom-up techniques consist of the growth of small particles from individual molecules. The driving force for the growth of a crystal from individual molecules is

H. de Waard $(\triangle) \cdot H$. W. Frijlink $\cdot$ W. L. J. Hinrichs

Department of Pharmaceutical Technology and Biopharmacy

University of Groningen

Antonius Deusinglaan I

9713 AV Groningen, The Netherlands

e-mail: h.de.waard@rug.nl supersaturation. Supersaturation of a drug in a solution can be obtained by decreasing the temperature or addition of an anti-solvent.

The size of crystals formed from supersaturated pure drug solutions depends on the balance between the nucleation rate and crystal growth. This balance between nucleation and growth is determined by the extent of supersaturation. At a higher extent of supersaturation, the nucleation rate increases, and, hence, the crystal size decreases, whereas at a lower extent of supersaturation, the growth rate increases, and, hence, the crystal size increases. Therefore, process conditions, such as temperature or the ratio between solvent and anti-solvent and their mixing rate, can be used to control the drug crystal size. When drug crystals are formed in drug composites, the size of the crystals is also determined by the interstitial spaces between matrix molecules.

While there are many excellent reviews on top-down techniques (e.g. (1,2)), bottom-up techniques are usually just referred to as "precipitation techniques." Bottom-up techniques can be considered as the oldest techniques to prepare drug nanocrystals. However, recently, interesting new developments in the field of bottom-up preparation of drug nanocrystals have evolved. This commentary focuses on these techniques. First, an overview of bottom-up preparation techniques of crystalline drug nanoparticles will be given (Table I). Second, the process-related barriers for products prepared by these techniques to reach the market will be discussed. For an introduction on top-down methods to prepare drug nanocrystals and the application thereof, the reader is referred to an excellent recently published review (2). 


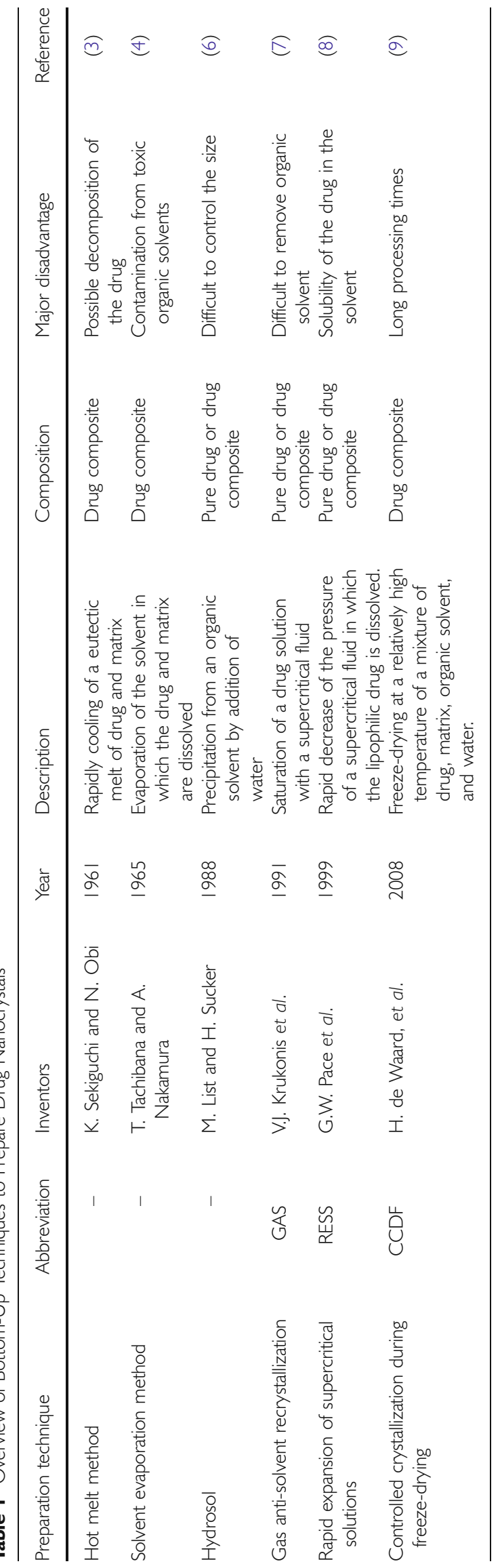

\section{BOTTOM-UP TECHNIQUES}

Since bottom-up techniques to prepare drug nanocrystals are commonly referred to as "precipitation methods," solid dispersions are usually not considered to be the first product containing drug nanocrystals. However, some types of solid dispersions consist of nanocrystalline drug particles embedded in a matrix. Already in the early 1960s, Sekiguchi et al. prepared a solid dispersion of the poorly soluble sulfathiazole embedded in urea (3). The small particles were prepared by melting the drug and matrix and subsequently cooling this mixture in an ice bath. This resulted in a carrier matrix with drug particles entrapped in it. The size of the nanoparticles could be controlled by process parameters such as the cooling rate.

A little later, Tachibani et al. developed the solvent evaporation method to prepare solid dispersions (4). To obtain a solid dispersion by this method, the drug and matrix are dissolved in a common solvent (e.g. chloroform), after which the solvent is evaporated under vacuum. When Tachibani developed this method, the drug was incorporated in the matrix monomolecularly or as amorphous nanoparticles. Although this can be a successful strategy to increase the dissolution rate, there are concerns regarding the physical stability of these solid dispersions. Since amorphous solid dispersions have the risk of uncontrolled crystallization during processing or storage, a crystalline product is usually preferred. After the solvent evaporation method was adjusted, it could be used to prepare nanocrystalline solid dispersions by this method as well (5).

The first traditional precipitation technique that has been developed is the so-called hydrosol technique, invented by List and Sucker (6). To obtain drug nanocrystals by this technique, the lipophilic drug is first dissolved in an organic solvent (e.g. ethanol) and then mixed with a large amount of anti-solvent that is miscible with the organic solvent, usually water. Mixing of the organic solution and the anti-solvent should be performed rapidly to assure fast nucleation and thereby small particles. To stabilize the nanocrystals, a carrier (e.g. gelatin) can be dissolved in the aqueous phase. Hereafter, the solvents can then be removed by evaporation or lyophilization to obtain a dry nanocrystalline drug product.

Another category of bottom-up techniques that was developed is commonly known as supercritical fluid technologies. Although there are currently many different processes to prepare drug nanocrystals based on supercritical fluid technologies, they are all more or less based on gas anti-solvent recrystallization (GAS) and rapid expansion of supercritical solutions (RESS). The application of GAS to obtain drug nanocrystals was for the first time described in 1991 by Krukonis et al. (7). In this process, the supercritical fluid acts as an anti-solvent. A solution of the lipophilic drug 
in an organic solvent is saturated with supercritical fluid (such as supercritical $\mathrm{CO}_{2}$ ), thereby decreasing the solubility of the drug in the solvent and consequently causing the drug to precipitate. The other supercritical fluid technique, RESS, was already widely described in other applications before it was for the first time described in 1999 by Pace et al. to prepare drug nanocrystals (8). In this process, the supercritical fluid acts as a solvent. The lipophilic drug is dissolved in the supercritical fluid, after which the pressure is rapidly decreased. Due to the decrease of solvent power, the drug rapidly precipitates from the supercritical fluid.

More recently, a freeze-drying technique to prepare drug nanocrystals, controlled crystallization during freezedrying (CCDF), was developed (9). This technology is based on freeze-drying a mixture of a non-toxic organic solvent (tertiary butyl alcohol) in which the drug is dissolved and an aqueous solution containing a matrix material (e.g. mannitol). Freeze-drying is performed at a relatively high temperature (above the glass transition temperature of the maximally freeze-concentrated fraction) to allow the drug and matrix to crystallize.

In the future, other bottom-up techniques to prepare drug nanocrystals based on existing processes may be developed. Bottom-up techniques, such as spray-freezing into liquid (10) or nanoprecipitation in microfluidic reactors (11), are promising techniques to prepare drug nanocrystals. Currently, they are used to prepare amorphous nanoparticles, but they may be adapted in such a way that they can be used to produce a nanocrystalline drug product. Spray-freezing into liquid (SFL), for example, consists of similar process steps as CCDF. A mixture of drug, polymer, organic solvent, and water is rapidly frozen and then freeze-dried. Although there are more differences between these two processes, the most important difference is that the solutions used during SFL are, at least at room temperature, thermodynamically stable, while the mixture used during GCDF is not. So far, SFL is only reported to yield amorphous nanoparticles, but when process conditions, such as the concentration organic solvent or the freeze-drying temperature are modified, crystalline nanoparticles may be obtained.

\section{BOTTOM-UP PREPARED NANOCRYSTALS ON THE MARKET}

Whereas there are already several nanocrystalline products on the market prepared by top-down methods (2), the number of products prepared by bottom-up methods is very limited. Rare examples are products such as GrisPEG $^{\circledR}$ (griseofulvin in PEG8000) and Cesamet ${ }^{\circledR}$ (nabilone in PVP). The few bottom-up prepared nanocrystalline products are still based on the solid dispersion technology as described by Sekiguchi and Obi, which dates back almost fifty years ago. Although there are many promising in-vitro results published, only a few of these efforts resulted in a marketed product.

It is therefore worthwhile to analyze why there are so few nanocrystalline drug products based on bottom-up preparation techniques. Commonly mentioned disadvantages of these techniques are possible decomposition of the drug (hot melt method), contamination from toxic organic solvents (solvent evaporation method), difficulty controlling the size of the drug crystals (hydrosol), limited solubility of the drug in the solvent (supercritical fluid technologies), and long processing times (CGDF). However, top-down methods also suffer from disadvantages such as contamination from grinding media, difficulty achieving a uniform size distribution, and long processing times. These disadvantages did not limit the development of commercialized nanocrystalline drug products based on top-down preparation techniques.

Therefore, it seems to be unlikely that the abovementioned disadvantages are the most important reasons that only a limited number of bottom-up prepared nanocrystalline drug products reached the market. A more plausible explanation may be the difficulty to scale-up the bottom-up processes. For obvious reasons, most bottompreparation methods are developed on lab-scale. However, to utilize these potentially useful bottom-up techniques, one should think about industrial production. This means that for each technique the possibility to change the process into a continuous and/or large scale process should be kept in mind. Also, the use of toxic organic solvents should be minimized to reduce the risks during manufacturing and the risk of high concentrations of residual impurities in the end product.

Large-scale production of drug nanocrystals by bottomup techniques is not necessarily problematic. Techniques such as the hot melt method, some supercritical fluid methods, and CCDF seem to be suitable for scale-up. For example, the lab-scale hot melt method can be changed into a large-scale holt melt extrusion process, and freezedrying during CCDF can be changed into continuous spray freeze-drying. However, more research should be performed on the actual feasibility of these techniques to be used for large-scale production, before they can be seen as an alternative to the currently used top-down methods.

In conclusion, although there are only a limited number of products based on bottom-up preparation of drug nanocrystals on the market, promising technologies are available. Examples of these techniques are hot melt extrusion, supercritical fluid technologies, and CCDF. However, products prepared by these or other bottom-up techniques will only reach the market if the industrial production is already kept in mind during lab-scale development. 
Open Access This article is distributed under the terms of the Creative Commons Attribution Noncommercial License which permits any noncommercial use, distribution, and reproduction in any medium, provided the original author(s) and source are credited.

\section{REFERENCES}

1. Rabinow BE. Nanosuspensions in drug delivery. Nature Rev. 2004;3:785-96.

2. Shegokar R, Müller RH. Nanocrystals: Industrially feasible multifunctional formulation technology for poorly soluble actives. Int J Pharm. 2010;399:129-39.

3. Sekiguchi K, Obi N. Studies on absorption of eutectic mixtures. I. A comparison of the behavior of eutectic mixtures of sulphathiazole and that of ordinary sulphathiazole in man. Chem Pharm Bull. 1961;9:866-72.
4. Tachibana T, Nakamura A. A methode for preparing an aqueous colloidal dispersion of organic materials by using water-soluble polymers: Dispersion of $\beta$-carotene by polyvinylpyrrolidone. Kolloid-Z Polym. 1965;203:130-3.

5. Leuner C, Dressman J. Improving drug solubility for oral delivery using solid dispersions. Eur J Pharm Biopharm. 2000;50:47-60.

6. List M, Sucker, H. Pharmaceutical colloidal hydrosols for injection. GB Patent 1988;2200048.

7. Krukonis VJ, Gallagher PM, Coffey MP. Gas anti-solvent crystallization process. US (1991);5:360-478.

8. Pace GW et al. Processes to generate submicron particles of waterinsoluble compounds. WO 1999;99/65469.

9. de Waard H, Hinrichs WLJ, Frijlink HW. A novel bottom-up process to produce drug nanocrystals: controlled crystallization during freeze drying. J Control Release. 2008;128:179-83.

10. Hu J et al. Improvement of dissolution rates of poorly water soluble APIs using novel spray freezing into liquid technology. Pharm Res. 2002;19:1278-84.

11. Ali HSM, York P, Blagden N. Preparation of hydrocortisone nanosuspension through a bottom-up nanoprecipitation technique using microfluidic reactors. Int J Pharm. 2009;375:107-13. 